\title{
IMPACTO DA ATIVIDADE FÍSICA COM AUXÍLIO DO EXERGAME NA SÍNDROME METABÓLICA EM ADOLESCENTES COM SOBREPESO OU OBESIDADE
}

\author{
IMPACT OF PHYSICAL ACTIVITY PERFORMED WITH EXERGAME \\ ON METABOLIC SYNDROME IN OVERWEIGHT OR OBESE \\ ADOLESCENTS
}

\author{
Morgana Monteiro Pimentel ${ }^{1}$ \\ Fernanda Caroline Tavares de Melo $^{2}$ \\ Geisielly Raquel da Cruz Aguiar ${ }^{3}$ \\ Gabrielle Sousa Marques ${ }^{4}$ \\ Carla Campos Muniz Medeiros ${ }^{5}$ \\ Danielle Franklin de Carvalho ${ }^{6}$
}

RESUMO: OBJETIVO: avaliar o impacto da atividade física, realizada com auxílio do exergame, na ocorrência de síndrome metabólica em adolescentes com sobrepeso ou obesidade. MÉTODOS: estudo de intervenção quase experimental, realizado com 48 adolescentes (15 a 19 anos) com sobrepeso ou obesidade matriculados em escolas públicas de ensino médio do município de Campina Grande - PB. Para a intervenção foi utilizada a plataforma XBOX 360, com acessório Kinect (Microsoft ${ }^{\circledR}$ ) e o Just Dance foi o jogo selecionado. Os dados foram analisados através do SPSS versão 22.0, e para todas as análises foi adotado um nível de significância de 5\%. RESULTADOS: no início do estudo, 63,0\% foram classificados como muito ativos ou ativos, $25,9 \%$ como insuficientemente ativos e $11,1 \%$ como sedentários. Após a intervenção, diminuiu de cinco $(10,4 \%)$ para três $(6,2 \%)$ o número de adolescentes com SM, redução não estatisticamente significante. CONCLUSÃO: os resultados indicaram que apesar do aumento do nível de atividade física proporcionado pela intervenção, a utilização do exergame

\footnotetext{
${ }^{1}$ Graduada em Enfermagem. Universidade Estadual da Paraíba, Campina Grande, Paraíba, Brasil.

${ }^{2}$ Graduada em Enfermagem. Universidade Estadual da Paraíba, Campina Grande, Paraíba, Brasil.

${ }^{3}$ Graduada em Enfermagem. Universidade Estadual da Paraíba, Campina Grande, Paraíba, Brasil.

${ }^{4}$ Graduada em Fisioterapia. Universidade Estadual da Paraíba, Campina Grande, Paraíba, Brasil.

5 Doutora em Saúde da Criança e do Adolescente. Universidade Estadual da Paraíba, Campina Grande, Paraíba, Brasil.

${ }^{6}$ Doutora em Saúde da Criança e do Adolescente. Universidade Estadual da Paraíba, Campina Grande, Paraíba, Brasil.
} 
não resultou em impacto estatisticamente significativo sobre a ocorrência de síndrome metabólica.

Palavras chave: Adolescentes; Obesidade; Síndrome Metabólica; Atividade Física.

ABSTRACT: Objective: to evaluate the impact of physical activity performed with exergame, on the occurrence of metabolic syndrome in overweight or obese adolescents. Methods: an almost experimental intervention study with 48 adolescents (15 to 19 years old) who were overweight or obese, enrolled in public high schools in the city of Campina Grande - PB. For theintervention, it was used the XBOX 360 platform, with Kinect accessory (Microsoft $\left.{ }^{\circledR}\right)$ and Just Dance was the game selected. Data were analyzed through SPSS version 22.0, and a significance level of 5\% was adopted for all analyzes. Results: at the beginning of the study, $63.0 \%$ were classified as very active or active, $25.9 \%$ as insufficiently active and $11.1 \%$ as sedentary. After the intervention, the number of adolescents with MS declined from five (10.4\%) to three (6.2\%), a non-statistically significant reduction. Conclusion: the results indicated that despite the increase in the level of physical activity provided by the intervention, the use of exergame did not result in a statistically significant impact on the occurrence of metabolic syndrome.

Keywords: Adolescents; Obesity; Metabolic syndrome; physical activity. 


\section{INTRODUÇÃO}

Atualmente a obesidade tem sido considerada um problema de saúde pública, pois a sua prevalência tem crescido a níveis alarmantes e vem ganhando destaque no cenário epidemiológico mundial (FREITAS et al., 2014). A Organização Mundial de Saúde (OMS) ressalta que o excesso de peso é fator de risco para o desenvolvimento de muitas doenças crônicas, tais como doenças cardiovasculares, diabetes e o câncer, além de desordens metabólicas, afetando, sem distinção, todas as faixas etárias (WANNMACHER, 2016).

A síndrome metabólica (SM) corresponde a presença de alterações glicídicas e lipídicas, da obesidade abdominal, da hipertensão arterial (HA) e de distúrbios da coagulação. Essas características representam um conjunto de fatores de risco cardiovascular (BARBOSA et al., 2010).

Estudo transversal, de âmbito nacional, realizado com escolares com idade entre 12 e 17 anos, revelou que aproximadamente 2,6\% de adolescentes brasileiros, de acordo com avaliação seguindo os critérios estabelecidos pela International Diabetes Federation (IDF), convivem com a SM, sendo ainda mais prevalente entre os indivíduos do sexo masculino (KUSCHNIR et al., 2016).

Alguns autores adotam a perspectiva do NCEP-ATPIII e afirmam que é portador desta síndrome aquele indivíduo que apresentar ao menos três dos seguintes critérios: hipertensão arterial, diabetes mellitus, hipertrigliceridemia, baixos níveis de HDL-colesterol e aumento da circunferência abdominal (CA) (FONOFF et al., 2015; CARVALHO et al., 2016).

O exercício físico tem sido apontado como terapêutica indispensável, por atuar de forma direta e indireta no combate aos fatores de risco que compõem a síndrome (VASCONCELOS et al., 2013). Encontrar atividades físicas que motivem os adolescentes a praticá-las de forma ativa e frequente é um desafio, entretanto, tornase essencial para promover a perda de peso ou a manutenção do peso saudável Diante disso, tecnologias de jogos de realidade virtual estão sendo aplicadas à 
saúde, podendo ser denominadas como exergames e têm servido como instrumento para aumentar o interesse do adolescente à prática de exercícios físicos.

O exergame corresponde a uma nova tecnologia que utiliza jogos visando a promoção de uma interação entre o jogador e imagens que são apresentadas na tela, através da realização de movimentos corporais, proporcionando ao indivíduo um aumento da prática de exercício físico e podendo gerar benefícios para a sua saúde (ZENG; GAO, 2016).

O desenvolvimento de novas tecnologias tem resultado em novas estratégias para a recuperação da saúde, gerando um impacto positivo e possibilitando maior disponibilidade de informações a respeito da promoção da saúde.

Diante do exposto, o presente estudo objetiva avaliar o impacto do exercício físico, realizado com auxílio do exergame, na ocorrência de síndrome metabólica em adolescentes com sobrepeso ou obesidade no município de Campina Grande - PB.

\section{MÉTODOS}

Trata-se de um estudo de intervenção quase-experimental, realizado no período de junho a dezembro de 2016. A amostra foi composta por 48 adolescentes com sobrepeso ou obesidade matriculados em escolas públicas de ensino médio de grande porte, do município de Campina Grande, Paraíba, com faixa etária entre 15 e 19 anos.

Para participar da pesquisa o adolescente devia estar regularmente matriculado em escolas de grande porte da rede pública de ensino, da zona urbana de Campina Grande-PB e cursando o primeiro ou o segundo ano do ensino médio nas escolas selecionadas; estar dentro da faixa etária determinada; apresentar excesso de peso corporal. Todavia, foram excluídos aqueles indivíduos que apresentassem alguma limitação motora ou mental que impedisse a participação nas atividades da intervenção e/ou realização das provas de função pulmonar; possuir alguma alteração metabólica severa que exigisse o uso de medicamentos ou tratamento específico que alterasse o metabolismo do perfil lipídico; possuir asma 
com crise recente (dois meses anteriores à coleta de dados) e/ou relato de broncoespasmo induzido pelo exercício; ser usuário de videogame ativo; e gestantes. No caso de desenvolvimento de algum dos critérios de exclusão ao longo do estudo, isso implicaria no desligamento do adolescente da pesquisa.

Para o desenvolvimento desta pesquisa foram utilizados os seguintes instrumentos de avaliação:

Ficha de avaliação das variáveis sociodemográficas: idade, em anos completos; sexo, masculino ou feminino; cor, autorreferida como branca, parda, preta, indígena ou amarela; e classe econômica, identificada pelo critério brasileiro de classificação (ABEP, 2010).

Os dados antropométricos (peso, altura e circunferência abdominal) foram coletados em duplicata, sendo considerado o valor médio das duas aferições. Para obtenção do peso foi utilizada uma balança digital Tanita ${ }^{\circledR}$, com capacidade para 150 $\mathrm{kg}$ e precisão de $0,1 \mathrm{~kg}$. A altura foi aferida através de um estadiômetro portátil da marca Tonelli ${ }^{\circledR}$, com precisão de $0,1 \mathrm{~cm}$. A CA foi determinada por medidas no ponto médio entre a última costela e a crista ilíaca, utilizando a fita métrica inelástica da marca Cardiomed $^{\circledR}$, com precisão de $0,1 \mathrm{~cm}$. O índice de massa corporal (IMC) foi determinado pela razão do peso, em $\mathrm{Kg}$, pelo quadrado da altura, em metros.

Para aferição da pressão arterial foram utilizados aparelhos semiautomáticos OMRON-HEM 705 CP. Foram seguidas as recomendações relatadas na VI Diretriz Brasileira de Hipertensão Arterial.

As dosagens bioquímicas foram realizadas sempre no período da manhã, observando o jejum de 12 horas, em laboratório de análises clínicas terceirizado, com selo de controle de qualidade ControlLab - Proficiência em Ensaios Laboratoriais (PELM). O colesterol total (CT), HDL-colesterol, triglicerídeos (TG) e glicemia de jejum (GJ) foram avaliados pelo método colorimétrico enzimático (equipamento automático BioSystems $310^{\circledR}$ ), de acordo com as recomendações do fabricante do kit Labtest $^{\circledR}$.

O diagnóstico da SM foi realizado através dos critérios recomendados pela National Cholesterol Education Program Adult Treatment Pannel III (NCEP/ATPIII, 2001), adaptados para faixa etária, que considera a presença de pelo menos três destes critérios: $C A \geq$ percentil 90 para sexo, idade e raça; triglicerídeos $\geq 100$ 
$\mathrm{mg} / \mathrm{dL} ; \mathrm{HDL}-\mathrm{c}<40 \mathrm{mg} / \mathrm{dL}$, GJ $\geq 100 \mathrm{mg} / \mathrm{dL}$ (seguindo as recomendações da American Diabetes Association - ADA), pressão sistólica e/ou diastólica acima ou no percentil 90 para o sexo, estatura e idade.

O estado nutricional foi classificado através do Índice de Massa Corporal (IMC), de acordo com as recomendações da Organização Mundial de Saúde (OMS), considerando-se sobrepeso (IMC $\geq+1$ e $<$ Escore-Z +2 ), obesidade IMC $\geq$ Escore-Z +2 e IMC < Escore- Z+3), obesidade acentuada (IMC $\geq$ Escore- $Z+3)$, sendo que, para os maiores de 18 anos $\left(\mathrm{kg} / \mathrm{m}^{2}\right.$ ) foi considerado: sobrepeso (IMC $\geq 25,0$ e $<30,0$ ), obesidade (IMC $\geq 30,0$ ) (CONDE; MONTEIRO, 2006; ONIS et al., 2007).

O nível de atividade física foi avaliado pelo "Questionário Internacional de Atividade Física" (IPAQ), versão curta. O IPAQ é um questionário desenvolvido pela OMS e pelo Centro de Controle e Prevenção de Doenças, que avalia o tipo de atividade física adotado em um certo período de tempo (últimos sete dias) (SAUCEDO-MOLINA et al., 2015).

A classificação da atividade física por esse instrumento baseia-se no Centro de Estudos do Laboratório de Aptidão Física de São Caetano do Sul (CELAFISCS), que subdividiu em cinco categorias: muito ativo, ativo, irregularmente ativo $A$, irregularmente ativo $B$ e sedentário. Para efeitos de análise de dados, foi realizada uma reclassificação em dois grupos: "ativos" (muito ativo e ativo) e "não ativos" (irregularmente ativo $A$, irregularmente ativo $B$ e sedentário). Esse questionário é validado para a utilização em adolescentes (HONG et al., 2012).

As escolas foram selecionadas por conveniência, de acordo com a maior prevalência de adolescentes com excesso de peso identificada em estudo anterior (RAMOS et al., 2016). As turmas selecionadas foram visitadas e foram explicados os procedimentos de pesquisa aos adolescentes, que receberam os esclarecimentos e o Termo de Consentimento Livre e Esclarecido (TCLE). Posteriormente, a escola foi visitada novamente para recolhimento dos termos. Foi realizado um check list para avaliar as condições de inclusão/exclusão no estudo. Em sequência foi realizada a triagem dos indivíduos, que, desta forma, compuseram a amostra. O nível de atividade física e os critérios para diagnóstico da Síndrome Metabólica foram avaliados no período pré e pós intervenção. 
A intervenção foi realizada com exergame através da prática de exercícios com auxílio de um videogame ativo e foi supervisionada e monitorizada. A intensidade foi moderada, frequência semanal de três vezes (nas escolas) e duração de 50 minutos cada sessão, por um período de oito semanas. Os jogos foram disponibilizados em salas destinadas para tal finalidade nas escolas selecionadas, em horários nos turnos da manhã e tarde.

Foi utilizada a plataforma XBOX 360, com acessório Kinect (Microsoft ${ }^{\circledR}$ ) e o Just Dance foi o jogo selecionado, pois além de atender ao critério de intensidade do exercício acima elencado, é relatado na literatura como o que desperta maior interesse entre os adolescentes (PEREIRA et al., 2012; BIDDISS; IRWIN, 2010).

A análise dos dados foi apresentada inicialmente com a estatística descritiva através da frequência absoluta e relativa para caracterização da população. Para avaliar o impacto do uso do videogame ativo com o nível de atividade física e a ocorrência de SM, foi utilizado o teste de McNemar. Os dados foram analisados através do SPSS versão 22.0. Para todas as análises foi adotado um nível de significância de $5 \%$.

A pesquisa foi desenvolvida de acordo com a Resolução N466/2012 do Conselho Nacional de Saúde, aprovada pelo Comitê de Ética em Pesquisa da Universidade Estadual da Paraíba (CAAE: 56118616.1.0000.5187) em 30 de maio de 2016.

\section{RESULTADOS}

Foram avaliados no estudo 55 adolescentes, dos quais 48 foram incluídos nos dois momentos (antes e depois da intervenção), perfazendo uma perda de 12,7\% $(n=7)$. Do total de 48,60,4\% $(n=29)$ era do sexo feminino, 56,3\% $(n=27)$ pertenciam às classes econômicas C, D e $E$, e 79,2\% (n=38) se autorreferiram como sendo de cor não branca. Com relação ao estado nutricional, 70,8\% $(n=34)$ tinham sobrepeso. A idade média foi de 16,3 \pm 1 ano. 
Quando avaliado o nível de atividade física no início do estudo, 24,1\% ( $n=12)$ foram classificados como muito ativos, 38,9\% $(n=19)$ como ativos, $14,8 \%(n=7)$ como insuficientemente ativos $A, 11,1 \%(n=5)$ como insuficientemente ativos $B$ e $11,1 \%$ $(n=5)$ como sedentários. Para avaliar o efeito da intervenção, a classificação foi categorizada em dois grupos: ativos (ativos e muito ativos) e inativos (insuficientemente ativos $A$, insuficientemente ativos $B$ e sedentários). Inicialmente aproximadamente $62,5 \%(n=30)$ da amostra era composta de indivíduos ativos. Conforme esperado, observou-se que a intervenção aumentou significativamente o nível de atividade física entre os adolescentes com sobrepeso ou obesidade, resultando em um total de $81,25 \%(n=39)$ de indivíduos ativos (Tabela 1$)$.

Tabela 1 - Distribuição dos 48 adolescentes escolares quanto ao nível de atividade física, avaliado pelo IPAQ, antes e depois da intervenção. Campina Grande- PB, 2017.

\begin{tabular}{|c|c|c|c|c|c|}
\hline \multirow{3}{*}{ Antes da intervenção } & \multicolumn{4}{|c|}{ Depois da intervenção } & \multirow{2}{*}{$P$} \\
\hline & \multicolumn{2}{|c|}{ Não ativos } & \multicolumn{2}{|c|}{ Ativos } & \\
\hline & $\mathrm{n}$ & $\%$ & $\mathrm{n}$ & $\%$ & \\
\hline Não ativos & 07 & 38,9 & 11 & 61,1 & $0,022^{*}$ \\
\hline Ativos & 02 & 6,7 & 28 & 93,3 & \\
\hline
\end{tabular}

Verificou-se que inicialmente cinco $(10,4 \%)$ adolescentes apresentaram SM e, após a intervenção, houve um total de três $(6,2 \%)$ com SM. Apesar da diferença matemática, não se pode afirmar que essa redução seja estatisticamente significante $(p=0,625)$ (Tabela 2$)$.

Tabela 2 - Distribuição dos 48 adolescentes escolares quanto à ocorrência de síndrome metabólica, antes e depois da intervenção. Campina Grande- PB, 2017.

\begin{tabular}{|c|c|c|c|c|c|}
\hline \multirow{3}{*}{ Antes da intervenção } & \multicolumn{4}{|c|}{ Depois da intervenção } & \multirow{2}{*}{$P$} \\
\hline & & & & & \\
\hline & $\mathrm{n}$ & $\%$ & $\mathrm{n}$ & $\%$ & \\
\hline Com SM & 02 & 40,0 & 03 & 60,0 & 0,625 \\
\hline Sem SM & 01 & 2,3 & 42 & 97,7 & \\
\hline
\end{tabular}




\section{DISCUSSÃO}

Neste estudo verificou-se uma maior prevalência de indivíduos do sexo feminino (60,4\%). Cabrera et. al. (2014) obteve resultado semelhante em seu estudo transversal realizado com crianças e adolescentes, revelando maior prevalência de sobrepeso relacionado ao sexo feminino. Não se verificou, entretanto, nenhuma relação dos resultados desse estudo quanto ao sexo.

Observou-se uma prevalência de indivíduos com excesso de peso entre as classes econômicas mais baixas. Resultado que difere ao encontrado por Christofaro et al. (2011) em seu estudo transversal, evidenciando excesso de peso mais associado aos adolescentes de classes econômicas mais altas.

Castro et al. (2018) em seu estudo descritivo com delineamento transversal, realizado com adolescentes que cursavam o ensino médio em uma escola pública, observou que entre os indivíduos com excesso de peso, houve maior índice de sobrepeso $(22,9 \%)$ a obesidade $(1,4 \%)$, resultado semelhante ao encontrado por nosso estudo.

A amostra desse estudo foi composta por uma maioria de indivíduos ativos. Entretanto, dentre os adolescentes participantes que não eram ativos, 11 passaram a ser ativos após o período de intervenção com o exergame, o que evidencia a sua eficácia sobre o nível de atividade física. Pesquisa como a de Shayne et al. (2012) realizou intervenção semelhante, apontando que houve melhora significativa no nível de atividade física, além de redução do excesso de peso.

Foi observada uma redução de dois casos da SM no período pós-intervenção com o exercício aeróbico com auxílio do exergame, entretanto, o impacto não foi considerado estatisticamente significante. Esse resultado pode ser atribuído ao curto período de tempo para realização do programa de exercícios, ao pequeno tamanho da amostra e também ao fato de que, embora se esteja trabalhando com uma população com excesso de peso, ela é constituída por indivíduos jovens e considerados saudáveis. 
No nosso estudo os adolescentes participavam de um programa de exercícios aeróbicos moderados com duração de 50 minutos por sessão. Levantamento realizado por Escalante et al. (2012) relatou que programas de exercícios aeróbicos realizados por uma média de 60 minutos, três vezes por semana, podem reduzir as concentrações de lipoproteínas de baixa densidade (LDL) e de triglicerídeos, além de aumentar as lipoproteínas de alta densidade (HDL).

Estudos que observaram redução da pressão arterial (PA), fator que também pode compor a SM, em adolescentes obesos com o treinamento físico, utilizaram exercícios aeróbios de três a seis vezes por semana, com duração entre 50 a 90 minutos, intensidade entre 55 a $75 \%$ da frequência cardíaca (FC) máxima e período de treinamento de 12 a 24 semanas (FARAH et al., 2012) o que implica em uma carga de exercício superior à realizada nesta pesquisa.

Já o estudo realizado por Colombo et al. (2013) avaliou os efeitos de um programa de exercícios de intensidade moderada em pacientes adultos com SM. Os indivíduos participaram de um programa com duração de 40 a 50 minutos de caminhada, três vezes por semana, durante 12 semanas. Ao fim do programa, observou-se uma redução na circunferência da cintura, dos níveis pressóricos e do IMC e melhora nos níveis de HDL-colesterol. Acredita-se que a diferença encontrada esteja mais relacionada ao tempo total de intervenção do que à classificação etária dos sujeitos, sugerindo uma ampliação do tempo de intervenção em estudos futuros.

\section{CONSIDERAÇÕES FINAIS}

Diante da interpretação dos resultados é possível afirmar que a utilização do exergame como ferramenta para o aumento do nível de atividade física foi eficaz. Quanto à síndrome metabólica, houve redução do número de casos após o programa de exercícios, porém o resultado não se mostrou estatisticamente significante.

Consideram-se fatores para limitação desse estudo o tempo estipulado para a intervenção, o tamanho da amostra, a não observação dos componentes da SM 
individualmente e dos hábitos alimentares. Esse estudo sugere que a utilização do exergame pode ser útil para o tratamento de indivíduos acometidos por esta síndrome e fornece a base para pesquisas futuras.

\section{DECLARAÇÃO DE CONFLITO DE INTERESSE}

Artigo fruto de um trabalho de conclusão de curso apresentado ao curso de Bacharelado em Enfermagem da Universidade Estadual da Paraíba (UEPB).

\section{FONTE FINANCIADORA}

Este trabalho recebeu financiamento da Universidade Estadual da Paraíba PROPESQ 2017. [Processo:4.06.01.00-5-415/2017-1].

\section{REFERÊNCIAS BIBLIOGRÁFICAS}

ABEP. Associação Brasileira de Empresas de Pesquisa. CCEB: Critério de Classificação Econômica Brasil. São Paulo, 2010.

BARBOSA, J. B. et al. Síndrome Metabólica em Ambulatório Cardiológico. Arq Bras Cardiol, v. 94, n. $1, \quad$ p. 46-54, 2010. Disponivel em: < http://www.scielo.br/scielo.php?script=sci_arttext\&pid=S0066-782X2010000100009 >. Acesso em 06 janeiro 2020.

BIDDISS, E.; IRWIN, J. Active Video Games to Promote Physical Activity in Children and Youth. Arch Periatr Adolesc Med, v.164, n.7, p.664-672, 2010. Disponível em: < https://www.ncbi.nlm.nih.gov/pubmed/20603468 >. Acesso em: 19 jul 2018.

CABRERA, T. F. et al. Análise da prevalência de sobrepeso e obesidade e do nível de atividade física em crianças e adolescentes de uma cidade do sudoeste de São Paulo. Journal of Human Growth and Development, v. 24, n. 1, 2014. Disponível em: < http://www.revistas.usp.br/jhgd/article/view/73455>. Acesso em: 18 jul 2018.

CARVALHO, R. B. N. et al. Fatores de risco associados ao desenvolvimento da síndrome metabólica em crianças e adolescentes. Acta. Paul. De Enferm, v. 29, n. 4, São Paulo, 2016. Disponível em: < http://www.scielo.br/pdf/ape/v29n4/1982-0194-ape-29-04-0439.pdf>. 
Acesso em: 17 jul 2018.

CASTRO, J. M. et al. Prevalência de sobrepeso e obesidade e os fatores de risco associados em adolescentes. Revista Brasileira de Obesidade, Nutrição e Emagrecimento, v. 12, n. 69, 2018. Disponível: < http://www.rbone.com.br/index.php/rbone/article/view/657/511 >. Acesso em: 16 nov. 2018.

CHRISTOFARO, D. G. D. et al. Prevalência de fatores de risco para doenças cardiovasculares entre escolares em Londrina - PR: diferenças entre classes econômicas. Revista Brasileira de Epidemiologia, v. 14, n. 1, São Paulo, 2011. Disponível em: < http://www.scielo.br/scielo.php?script=sci_arttext\&pid=S1415-790X2011000100003 >. Acesso em: 14 out 2018.

COLOMBO, C. M. Efeitos de curto prazo de um programa de atividade física moderada em pacientes com síndrome metabólica. Revista Einstein, v. 11, n. 3, São Paulo, 2013. Disponível em: < http://www.scielo.br/pdf/eins/v11n3/a11v11n3.pdf >. Acesso em: 05 setembro 2018.

CONDE, W. L., MONTEIRO, C. A. Valores críticos do índice de massa corporal para classificação do estado nutricional de crianças e adolescentes brasileiros. Jornal de Pediatria, v. 82, n. 4, São Paulo, 2006. Disponível em: < http://www.scielo.br/pdf/jped/v82n4/v82n4a07 >. Acesso em: 30 outubro 2018.

ESCALANTE, Y. et al. Improvement of the lipid profile with exercise in obese children: a systematic review. Preventive Medicine, v. 54, p. 293-301, 2012. Disponível em: < https://www.sciencedirect.com/science/article/pii/S0091743512000497 >. Acesso em 05 setembro 2018.

FARAH, B. Q. et al. Efeito do treinamento físico na pressão arterial de adolescentes com obesidade. Revista Paulista de Pediatria, v. 30, n. 4, p. 600-607, São Paulo, 2012. Disponível em: < http://www.scielo.br/pdf/rpp/v30n4/20.pdf >. Acesso em 05 setembro 2018.

FONOFF, J. et al. Prevalência de Síndrome Metabólica em adolescentes obesos: critérios diagnósticos. CuidArte Enfermagem, v. 9, n. 1, p. 78-84, São Paulo, 2015. Disponível em: < http://fundacaopadrealbino.org.br/facfipa/ner/pdf/Revistacuidarteenfermagem $\% 20 \mathrm{v} . \% 209 \% 2$ On. 1\%20\%20jan.\%20jun\%202015.pdf >. Acesso em 28 agosto 2018.

FREITAS, L. K. P. et al. Obesidade em adolescentes e as políticas públicas de nutrição. Ciênc. saúde colet, v. 19, n. 6 , p. 1755-1762, Junho, 2014. Disponível em: < http://www.scielo.br/scielo.php?script=sci_arttext\&pid $=\$ 1413-81232014000601755>$. Acesso em 06 janeiro 2020.

HONG, T. K. et al. Validity and reliability of a physical activity questionnaire for Vietnamese adolescents. International Journal of Behavioral Nutrition and Physical Activity, v. 9, n. 1, p. 93, 2012. Disponível em: < https://www.ncbi.nlm.nih.gov/pubmed/22853177 >. Acesso em: 22 jul. 2018.

KUSCHNIR, M. C. et al. ERICA: prevalência de síndrome metabólica em adolescentes brasileiros. Rev. Saúde Pública, v. 50, supl. 1, São Paulo, 2016. Disponível em: <http://www.scielo.br/pdf/rsp/v50s1/pt_0034-8910-rsp-S01518-87872016050006701.pdf>. Acesso em: 19 jul 2018.

NECP-ATP III. National Cholesterol Education Program (NCEP) Expert Panel on Detection, Evaluation, ad Treatment of High Blood Cholesterol in Adults (Adult Treatment Panel III): Executive Summary of the Third Report of The National Cholesterol Education Program (NCEP) Expert Panel on Detection, Evaluation, ad Treatment of High Blood Cholesterol In Adults (Adult Treatment Panel III). JAMA. 2001; 285: 2486-97.

ONIS, M. et al. Development of a WHO growth reference for school-aged children and 
adolescents. Bulletin of the World Health Organization, v. 85, n. 9, 2007. Disponível em: < https://www.scielosp.org/scielo.php?script=sci_arttext\&pid=S0042-

96862007000900010\&Ing=en\&nrm=iso\&tIng=en >. Acesso em: 30 outubro 2018.

PEREIRA, J. C. et al. Exergames como alternativa para o aumento do dispêndio energético: uma revisão sistemática. Revista Brasileira de Atividade Física e Saúde, v.17, n.5, p.327- 35 , 2012. Disponível em: < http://rbafs.org.br/RBAFS/article/view/2171>. Acesso em: 19 jul 2018.

RAMOS, T. D. A., DANTAS, T. M. E., SIMÕES, M. O. S., CARVALHO, D. F., MEDEIROS, C. C. Assessment of the carotid artery intima-media complex through ultrasonography and the relationship whit pathobiological determinants of atherosclerosis in youth. Cardiology in the Young, v. 26, p. 1333-1342, 2016. Disponivel em: < https://www.cambridge.org/core/journals/cardiology-in-the-young/article/assessment-of-the- carotidartery-intimamedia-complex-through- ultrasonography-and-the-relationship-withpathobiological-determinants-of-atherosclerosis-in-

youth/27891CCD4C585F5ADDF485476CAAF795 >. Acesso em: 30 outubro 2018.

SAUCEDO-MOLINA, T.; RODRÍGUEZ, J.; OLIVA, L.; et al. Relacion entre el indice de masa corporal, la actividad fisica y los tiempos de comida en adolescentes mexicanos. Nutr Hosp, $v$. 32, n. 3, p. 1082-90, 2015. Disponível em: <http://www.aulamedica.es/nh/pdf/9331.pdf>. Acesso em: 27 jul 2018.

SHAYNE, R. K., FOGEL, V. A., KOEHLER, S. The effects of exergaming on physical activity in a third-grade physical education class. Journal of Applied Behavior Analysis, v. 45, n. 1, 2012. Disponível em: < https://www.ncbi.nlm.nih.gov/pmc/articles/PMC3297345/ >. Acesso em: 29 out 2018.

Sociedade Brasileira de Cardiologia. Sociedade Brasileira de Hipertensão. Sociedade brasileira de Nefrologia. VI Diretrizes Brasileiras de Hipertensão. Arq Bras Cardiol. 2010;95(1):1-51.

VASCONCELLOS, F. V. A. et al. Exercício físico e síndrome metabólica. Revista HUPE, v.12, n. 4, p. 78-88, Rio de Janeiro, 2013. Disponível em: < http://revista.hupe.uerj.br/detalhe_artigo.asp?id=446>. Acesso em: 19 jul 2019.

WANNMACHER, L. Obesidade como fator de risco para morbidade e mortalidade: evidências sob o manejo com medidas não medicamentosas. OPAS/OMS, v. 1, n. 7, p. 1-10, Maio, 2016. Disponível em:

https://www.paho.org/bra/index.php?option=com_content\&view=article\&id=5134:novapublicacao-sobre-uso-racional-de-medicamentos-aborda-manejo-nao-medicamentoso-emcasos-de-obesidade-2\&ltemid=838 >. Acesso em 06 janeiro 2020.

ZENG, N. GAO. Z. Exergaming and obesity in youth: current perspectives. Int J Med, v. 9, p. 275-284, 2016. Disponível em: < https://www.ncbi.nlm.nih.gov/pmc/articles/PMC4977069/ >. Acesso em 28 agosto 2018. 